\title{
Patient care standards for primary mitochondrial disease: a consensus statement from the Mitochondrial Medicine Society
}

\author{
Sumit Parikh, MD ${ }^{1}$, Amy Goldstein, $\mathrm{MD}^{2}$, Amel Karaa, MD³ , Mary Kay Koenig, MD ${ }^{4}$, \\ Irina Anselm, MD ${ }^{5}$, Catherine Brunel-Guitton, MD, FRCPC ${ }^{6}$, John Christodoulou, MBBS, PhD ${ }^{7}$, \\ Bruce H. Cohen, MD ${ }^{8}$, David Dimmock, MD ${ }^{9}$, Gregory M. Enns, MB, ChB ${ }^{10}$, Marni J. Falk, MD ${ }^{11}$, \\ Annette Feigenbaum, MD ${ }^{12,13}$, Richard E. Frye, MD, PhD ${ }^{14}$, Jaya Ganesh, MD ${ }^{15}$, \\ David Griesemer, MD ${ }^{16}$, Richard Haas, MB BChir, MRCP ${ }^{17,18}$, Rita Horvath, MD, PhD ${ }^{19}$, \\ Mark Korson, MD ${ }^{20}$, 'Michael C. Kruer, MD21, Michelangelo Mancuso, MD, PhD ${ }^{22}$, \\ Shana McCormack, MD ${ }^{23}$, Marie Josee Raboisson, MD ${ }^{24}$, Tyler Reimschisel, MD, MHPE ${ }^{25}$, \\ Ramona Salvarinova, MD, FRCPC ${ }^{26}$, Russell P. Saneto, DO, PhD ${ }^{27}$, Fernando Scaglia, MD ${ }^{28}$, \\ John Shoffner, MD ${ }^{29}$, Peter W. Stacpoole, PhD, MD ${ }^{30}$, Carolyn M. Sue, MBBS, PhD ${ }^{31}$, \\ Mark Tarnopolsky, MD, PhD ${ }^{32}$, Clara Van Karnebeek, MD, PhD ${ }^{33,34}$, Lynne A. Wolfe, MS, CRNP ${ }^{35}$, \\ Zarazuela Zolkipli Cunningham, MBChB, MRCP ${ }^{36}$, Shamima Rahman, $\mathrm{FRCP}, \mathrm{PhD}^{37}$ and \\ Patrick F. Chinnery, FRCP, FMedSci ${ }^{38}$
}

The purpose of this statement is to provide consensus-based recommendations for optimal management and care for patients with primary mitochondrial disease. This statement is intended for physicians who are engaged in the diagnosis and management of these patients. Working group members were appointed by the Mitochondrial Medicine Society. The panel included members with several different areas of expertise. The panel members utilized surveys and the Delphi method to reach consensus. We anticipate that this statement will need to be updated as the field continues to evolve. Consensus-based recommendations are provided for the routine care and management of patients with primary genetic mitochondrial disease.

Genet Med advance online publication 27 July 2017

Key Words: care guidelines; consensus criteria; Delphi method; mitochondrial disease; treatment

\section{INTRODUCTION}

Primary genetic mitochondrial disorders arise from defects in either the mitochondrial or nuclear genome that impair mitochondrial function and structure. They are among the most common inborn errors of metabolism, with a minimum incidence of 1:5,000. Advances in genomics have allowed for improved diagnosis.

Multisystem disease manifestations often occur in mitochondrial patients leading to a need for comprehensive and

\footnotetext{
${ }^{1}$ Center for Child Neurology, Cleveland Clinic Children's Hospital, Cleveland, Ohio, USA; ${ }^{2}$ Division of Child Neurology, Children's Hospital of Pittsburgh, Pittsburgh, Pennsylvania, USA; ${ }^{3}$ Division of Genetics, Massachusetts General Hospital, Boston, Massachusetts, USA; ${ }^{4}$ Division of Child and Adolescent Neurology, University of Texas Medical School at Houston, Houston, Texas, USA; ${ }^{5}$ Department of Neurology, Boston Children's Hospital, Boston, Massachusetts, USA; ${ }^{6}$ Department of Pediatrics, University of Montreal, Montreal, Quebec, Canada; ${ }^{7}$ Neurodevelopmental Genomics Research Group, Murdoch Childrens Research Institute, and Department of Paediatrics, Melbourne Medical School, University of Melbourne, Melbourne, Australia; ${ }^{8}$ Neurodevelopmental Science Center, Children's Hospital Medical Center of Akron, Akron, Ohio, USA; ${ }^{9}$ Rady Children's Institute for Genomic Medicine, San Diego, California, USA; ${ }^{10}$ Division of Medical Genetics, Department of Pediatrics, Stanford University Lucile Packard Children's Hospital, Palo Alto, California, USA; ${ }^{11}$ Division of Human Genetics, Department of Pediatrics, The Children's Hospital of Philadelphia and University of Pennsylvania Perelman School of Medicine, Philadelphia, Pennsylvania, USA; ${ }^{12}$ Division of Clinical and Metabolic Genetics, The Hospital for Sick Children and University of Toronto, Toronto, Ontario, Canada; ${ }^{13}$ Department of Pediatrics, University of California San Diego and Rady Childrens Hospital, San Diego, California, USA; ${ }^{14}$ Department of Pediatrics, University of Arkansas Medical Sciences, Little Rock, Arkansas, USA; ${ }^{15}$ Division of Genetics, Department of Pediatrics, Cooper Medical School at Rowan University, Camden, New Jersey, USA; ${ }^{16}$ Division of Neurology, Levine Children's Hospital, Charlotte, North Carolina, USA; ${ }^{17}$ Departments of Neurosciences and Pediatrics, University of California San Diego, La Jolla, California, USA; ${ }^{18}$ Department of Neurosciences, Rady Children's Hospital, San Diego, California, USA; ${ }^{19}$ Institute of Genetic Medicine, Newcastle University, Newcastle upon Tyne, UK; ${ }^{20}$ Genetic Metabolic Center for Education, Salem, Massachusetts, USA; ${ }^{21}$ Department of Pediatric Neurology, University of Arizona College of Medicine, Phoenix, Arizona, USA; ${ }^{22}$ Department of Experimental and Clinical Medicine, Neurological Clinic, University of Pisa, Pisa, Italy; ${ }^{23}$ Division of Endocrinology and Diabetes, Children's Hospital of Philadelphia, Philadelphia, Pennsylvania, USA; ${ }^{24}$ Department of Cardiology, CHU Sainte-Justine, Montreal, Quebec, Canada; ${ }^{25}$ Department of Pediatrics, Vanderbilt University Medical Center, Nashville, Tennessee, USA; ${ }^{26}$ Division of Biochemical Diseases, BC Children's Hospital, British Columbia, Canada; ${ }^{27}$ Department of Neurology, Seattle Children's Hospital/University of Washington, Seattle, Washington, USA; ${ }^{28}$ Department of Molecular and Human Genetics, Baylor College of Medicine and Texas Children's Hospital, Houston, Texas, USA; ${ }^{29}$ Neurology, Biochemical \& Molecular Genetics, Atlanta, Georgia, USA; ${ }^{30}$ Department of Medicine, University of Florida College of Medicine, Gainesville, Florida, USA; ${ }^{31}$ Department of Neurology and Kolling Institute, Royal North Shore Hospital, St Leonards, Australia; ${ }^{32}$ Division of Neurology, McMaster University, Hamilton, Ontario, Canada; ${ }^{33}$ Department of Pediatrics, Academic Medical Centre, University of Amsterdam, Amsterdam, The Netherlands; ${ }^{34}$ Department of Pediatrics, Centre for Molecular Medicine, University of British Columbia, Vancouver, British Columbia, Canada; ${ }^{35}$ Undiagnosed Diseases Network, National Institutes of Health, Bethesda, Maryland, USA; ${ }^{36}$ Division of Neurology, The Children's Hospital of Philadelphia, Philadelphia, Pennsylvania, USA; ${ }^{37}$ Mitochondrial Research Group, UCL Great Ormond Street Institute of Child Health, London, UK; ${ }^{38}$ Department of Clinical Neurosciences \& MRC Mitochondrial Biology Unit, University of Cambridge, Cambridge, UK. Correspondence: Sumit Parikh (parikhs@ccf.org)
} 
multidisciplinary care. Mitochondrial specialty centers have formed over the past decade to meet this need, allowing the field of clinical mitochondrial medicine to develop. No published recommendations exist to guide patient management or clinical care decisions and standardize preventive health maintenance. Practice patterns vary considerably from center to center. ${ }^{1,2}$ The Mitochondrial Medicine Society (MMS) developed consensus criteria to aid with the diagnosis of mitochondrial disease. ${ }^{3}$ This prior publication addressed select management topics but was primarily focused on establishing diagnosis consensus guidelines.

An assessment completed by the MMS showed that $99 \%$ of 207 clinicians worldwide seeing mitochondrial patients (including neurologists, geneticists, and metabolic experts) were interested in having additional guidelines available for assisting in managing their care. More than half of these respondents (51\%) were aware of the Diagnostic Consensus Criteria previously published by the MMS, ${ }^{3}$ with $97 \%$ finding the guidelines useful and $82 \%$ stating that the recommendations directly impacted patient management.

This statement provides consensus-based recommendations for standardized treatment and preventive health care of patients with primary mitochondrial disease. For ease of reference and use, the recommendations are organized with respect to the various organ systems commonly involved in mitochondrial disorders, with sections on general symptoms and presentations. This new consensus statement expands upon and is intended to complement, but does not supplant, the 2015 consensus statement "Diagnosis and Management of Mitochondrial Disease."3 Importantly, our aim is not to provide an inflexible treatment protocol; the aim is to provide guidance based on an international consensus of experienced mitochondrial disease physicians.

In the interest of brevity, limited background information about the various mitochondrial disease manifestations and their comorbidities is provided here. We direct the reader who may be unfamiliar with these topics to review articles referenced within individual sections.

\section{METHODS}

Working group members were appointed by the Mitochondrial Medicine Society's Consensus Criteria Committee. An international panel included 35 mitochondrial medicine specialists-34 physicians and one nurse practitioner-from Australia (2), Canada (5), Italy (1), Netherlands (1), the United Kingdom (3), and the United States (23) with specialization in biochemical genetics, cardiology, clinical genetics, endocrinology, neurogenetics, or pediatric neurology. Our aim was to capture current clinical practice in these different countries and specialties.

Twenty organ- or system-specific clinician-led subgroups were formed, each with at least two members, to review relevant literature and create summaries on select symptoms and comorbid conditions in mitochondrial disease. Some members served on more than one subgroup when needed. Literature summaries were created via a PubMed Medical
Subject Heading and keyword search of organ- or systemspecific manifestations in mitochondrial disease. Primary literature and review articles were both reviewed for the working group summaries.

The field of mitochondrial medicine is not at a point where there is adequate high-level data to establish evidence-based clinical practice protocols. Prior classification of the level of evidence for treatment options in mitochondrial disease show that most publications are retrospective reports, case series, and nonblinded, nonrandomized trials. ${ }^{4,5}$ Thus, the panel developed recommendations using the Delphi method, a consensus method developed to utilize expert opinion to make a knowledge-based decision when insufficient information is available. ${ }^{6}$ This method is increasingly used to develop consensus-based guidelines in medicine and rare diseases. Expert panelists review and summarize the available knowledge and answer surveys concerning the issues in question. The survey is scored to determine the variation in opinion; if consensus is not reached, these items are returned to the panelists for a second round, this time with the mean of responses from the first round available. If needed, a meeting is then held to review areas of persistent disagreement.

The survey instrument was a five-point bipolar Likert scale; the range of potential responses included strongly disagree, disagree, neutral, agree, or strongly agree. Each subgroup was tasked with creating key clinical management recommendations to address within its focus area. Each response was assigned a numerical score, such that strongly disagree was scored as 1 , disagree was scored as 2 , neutral as 3 , agree as 4 , and strongly agree as 5 . The mean consensus score for each item was then tallied. As per the Delphi criteria, items with a mean consensus of $80 \%$ or more (score $>4$ (Agree/Strongly Agree) OR $<2$ (Disagree/Strongly Disagree)) were considered to reach consensus. Items that met criteria as recommendations did not necessarily have unanimous consensus.

All data summaries were reviewed and 25 surveys were completed by all authors; one for each set of organ-system recommendations and additional surveys for added recommendations by the working group and for those items that lacked consensus. The summaries and initial survey scores are available in the Supplementary Material online.

For items that did not meet consensus, a streamlined survey was returned to the panelists for a second round, with items marked with the group's score so that each panelist was aware of the group mean when they rescored the survey. Following completion of all surveys, a decision was reached by the working group to discard those recommendations that did not meet consensus. Only items that met consensus as defined by the Delphi process (scores $>4$ ) are included in the formal recommendations.

\section{RECOMMENDATIONS}

To orient the reader and contextualize recommendations, brief content synopses of various disease manifestations are provided. Recent published reviews on the topic are 


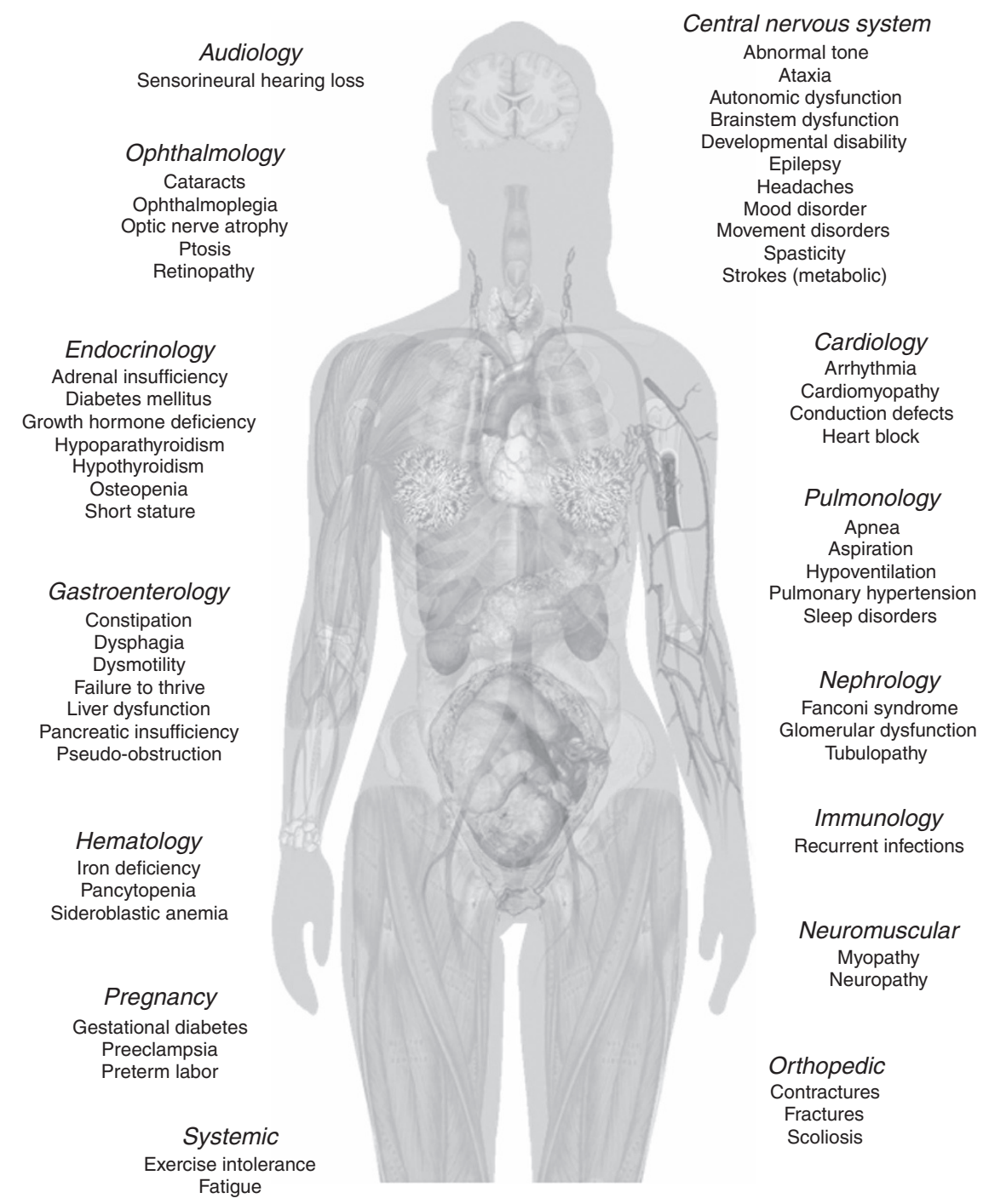

Figure 1 Body systems affected by primary mitochondrial disease.

referenced when available. Core topic areas are summarized in Figure 1 and presented in alphabetical order. A summary of recommended screening guidelines and specialist consultations to consider are placed in Tables 1 and 2. Previously published illness, anesthesia, and stroke management guidelines $^{3}$ are summarized in Table 3. A list of medications that need to be used with caution in mitochondrial patients is summarized in Table 4.

\section{Audiology}

Hearing loss is a frequent manifestation of mitochondrial disease though the prevalence is not yet known. A recent comprehensive review article on the topic is not available though some aspects were recently reviewed. ${ }^{7,8}$ Progressive sensorineural hearing loss, typically of cochlear origin, ${ }^{9}$ is the most common form, although congenital forms and auditory neuropathy can occur. Sensorineural hearing loss is believed to result mainly from reduced energy production within both stria vascularis and hair cells and increased reactive oxygen species leading to apoptosis. The health of both the auditory nerve and central auditory system are impacted by mitochondrial disease as well, especially when there is central nervous system (CNS) involvement. In addition, patients may have hearing loss caused, precipitated, or exacerbated by aminoglycosides, such as gentamicin. Sensorineural hearing loss usually affects the higher frequencies first, then progresses to involve the middle, and then lower frequencies. ${ }^{7,8}$

\section{Recommendations}

1. Formal audiology testing should be performed at time of diagnosis and then every $1-2$ years to monitor progression. A sensorineural or auditory neuropathy pattern of hearing loss should be confirmed.

2. Patients should be counseled to avoid environmental and other excessive noise exposure since this can exacerbate hearing loss and even lead to a step-wise deterioration in hearing ability. This exacerbation may or may not be reversible. 
Table 1 Screening guidelines

\begin{tabular}{|c|c|c|c|c|}
\hline & $\begin{array}{l}\text { At diagnosis, if } \\
\text { not previously } \\
\text { obtained }\end{array}$ & $\begin{array}{l}\text { At 1- to 2-year } \\
\text { intervals and } \\
\text { as needed }\end{array}$ & $\begin{array}{l}\text { As needed depending } \\
\text { on symptoms or } \\
\text { disease type }\end{array}$ & Comments \\
\hline Audiology & - & - & & \\
\hline Blood pressure & - & - & & \\
\hline Echocardiogram & $\bullet$ & $\bullet$ & & $\begin{array}{l}\text { Echocardiograms may be performed less frequently } \\
\text { in low-risk patients after several years of monitoring }\end{array}$ \\
\hline Holter & & & - & $\begin{array}{l}\text { Holter recording depending on the underlying } \\
\text { diagnosis and risk of heart block; up to every } \\
3-6 \text { months for disorders associated with high risk } \\
\text { of arrhythmias, such as mtDNA deletion disorders }\end{array}$ \\
\hline Cardiac MRI & & & $\bullet$ & \\
\hline Endocrinology & & & & $\begin{array}{l}\text { Endocrine screening strongly recommended in } \\
\text { those with mtDNA deletion disorders }\end{array}$ \\
\hline Ca and phosphate, urine & - & $\bullet$ & & \\
\hline Gonadotropins & & & • & \\
\hline Hemoglobin A1c & - & $\bullet$ & & \\
\hline Parathyroid hormone & - & & • & \\
\hline Thyroid-stimulating hormone and free thyroxine & $\bullet$ & $\bullet$ & & \\
\hline Vitamin D & - & - & & \\
\hline Dual X-ray Absorptiometry (DXA) & & & - & DXA especially if unexpected fractures \\
\hline \multicolumn{5}{|l|}{ Gastroenterology } \\
\hline Amylase-lipase & & & - & \\
\hline Transaminases & - & - & & \\
\hline \multicolumn{5}{|l|}{ Neurology } \\
\hline Developmental and cognitive assessments & $\bullet$ & • & & $\begin{array}{l}\text { Clinical appraisal or formal neuropsychological } \\
\text { tests; formal testing recommended with regression }\end{array}$ \\
\hline Electroencephalogram & & & - & \\
\hline \multicolumn{5}{|l|}{ Ophthalmology } \\
\hline Exam & - & - & & \\
\hline Electroretinogram & & & $\bullet$ & \\
\hline Optical coherence tomography & & & - & \\
\hline Psychiatry & $\bullet$ & - & & \\
\hline \multicolumn{5}{|l|}{ Mood and anxiety disorder screening } \\
\hline \multicolumn{5}{|l|}{ Pulmonology } \\
\hline Pulmonary function & & & - & $\begin{array}{l}\text { Especially with myopathy, if nonambulatory or with } \\
\text { brain stem dysfunction }\end{array}$ \\
\hline Polysomnogram & & & - & \\
\hline \multicolumn{5}{|l|}{ Renal } \\
\hline CMP with Mg and phosphate & • & • & & \\
\hline Albumin/creatine, urine & - & - & & \\
\hline
\end{tabular}

ACTH, adrenocorticotropic hormone; CMP, comprehensive metabolic panel; DXA, dual-energy X-ray; MRI, magnetic resonance imaging; mtDNA, mitochondrial DNA. 
Table 2 Other specialist consultations to consider at time of diagnosis and at 1-2 year intervals as needed based on symptoms

\begin{tabular}{l} 
Audiology \\
Cardiology \\
Endocrinology \\
Ear, nose, and throat \\
Gastroenterology \\
Genetics \\
Hematology \\
Immunology \\
Nephrology \\
Neurology \\
Ophthalmology \\
Orthopedics \\
Palliative care \\
Physical medicine and rehab/physiatry \\
Psychiatry (for patient or family) \\
Psychology (including family counseling) \\
Pulmonology \\
Social work \\
Sleep medicine \\
Therapy services including physical therapy, occupational therapy, and \\
speech therapy \\
\hline
\end{tabular}

3. Aminoglycosides may exacerbate or precipitate the development of hearing loss and should be used with caution.

4. Moderate to severe hearing loss should be treated with digital hearing aids to improve function; when profound, cochlear implantation should be considered.

\section{Cardiology}

The cardiac muscle is a high-energy-dependent tissue and both cardiomyopathy and arrhythmias are frequently encountered in mitochondrial diseases. A recent retrospective study of more than 200 patients with genetically confirmed mitochondrial DNA (mtDNA) or nuclear DNA mutations found cardiac involvement in $30 \%{ }^{10}$ Recent reviews of cardiac manifestations in primary mitochondrial disease have been published. ${ }^{11,12}$

The frequency of cardiomyopathy in patients with mitochondrial disease has been estimated to be $20-40 \%$ in older studies though not all had genetically confirmed disease. More recent work has reported cardiac anomalies such as impaired left ventricular ejection fraction, left ventricular hypertrophy, or late gadolinium enhancement in up to $53 \%$ of their cohort of patients with mitochondrial encephalopathy, lactic acidosis, and stroke (MELAS); chronic progressive external ophthalmoplegia; or Kearns-Sayre syndrome (KSS). ${ }^{13}$ While hypertrophic cardiomyopathy seems to be more prevalent, dilated, restrictive, left ventricular noncompaction and histiocytoid cardiomypathies have been described. ${ }^{12}$
Cardiac conduction abnormalities with an unpredictable course are also a frequent manifestation and arrhythmias are a common cause of death, particularly in KSS and those with the m.3243A > G mutation. Patients are at risk for sudden death or major cardiac event. In some studies cardiac conduction defects were reported in greater than $10 \%$ of patients with mitochondrial disease. ${ }^{10}$ Wolf-ParkinsonWhite syndrome, incomplete bundle branch or atrioventricular blocks, premature ventricular contractions, and preexcitation resulting in supraventricular arrhythmias have all been noted. ${ }^{14}$ Intraventricular conduction block is a significant independent risk factor for major adverse cardiac events in patients with mitochondrial disease. ${ }^{10}$

\section{Recommendations}

1. Care at a tertiary center with cardiology expertise in mitochondrial diseases is preferred.

2. Baseline assessments should include:

a. Standard 12-lead electrocardiogram (ECG) and echocardiogram (with measurement of left ventricular mass and systolic/diastolic function)

b. 24-hour ECG (Holter monitoring) in patients with palpitations, paroxysmal events, or in high-risk patients including patients with cardiomyopathy

c. Screening tests should include asymptomatic mutation carriers at significant risk of developing cardiac disease, especially those with maternally inherited mtDNA mutations known to be associated with cardiac disease

3. Follow-up evaluation should include clinical assessment, blood pressure measurement, ECG, and echocardiography every 12 months for at least 3 years.

a. Earlier follow-up is suggested for those with newonset cardiac symptoms or those at higher risk.

b. Extension of this 12-month interval should be considered only after discussion with a clinician experienced in the management of cardiac involvement; however, if the patient is stable for 3 years, ECG and echocardiography may be extended to every 2-3 years with return to yearly follow-up (or more frequently) if ECG or echocardiography suggests cardiac deterioration.

c. In asymptomatic mutation carriers, the interval of follow-up should be according to their risk of developing disease.

4. 24- to 48-hour ECG monitoring should be obtained every 1-2 years for all patients at high risk of preexcitation syndrome/conduction disease, patients with severely impaired left ventricular systolic function (left ventricular ejection-fraction $<35 \%$ ), patients with paroxysmal symptoms suggestive of cardiac involvement (palpitations), and patients with left ventricular systolic or diastolic dysfunction. 
Table 3 IIIness, anesthesia, and stroke management

IIIness management ${ }^{3}$

1. Specific decisions about patient management including hospitalization require clinical judgment and should be case-specific. Decisions should reflect the individual patient's presentation as well as an understanding of the etiology for the acute decompensation and the pathophysiology of the underlying mitochondrial disorder.

2. Patients with a mitochondrial disease should carry an emergency care plan that details their underlying disorder and provides management recommendations.

3. Patients with a mitochondrial disease should consider wearing a medical alert bracelet when appropriate depending on their clinical symptomology. 4. Mitochondrial patients should take precautions to prevent entering catabolism, especially when exposed to medical stressors, including avoiding prolonged fasting and receiving dextrose-containing IV fluids before, during, and after procedures and surgeries. (Dextrose should not be provided or provided in limited quantity as indicated by clinical status in suspected or confirmed disorders of pyruvate metabolism, if the patient is on a ketogenic diet, or the patient has had a previous adverse response to high glucose delivery.)

5. Evaluation of a mitochondrial patient in the acute setting should include evaluation of routine chemistries, glucose, transaminases, and lactate; all other testing is as clinically indicated, although one must keep in mind the potential for cardiac and neurologic decompensations in these patients. 6. Treatment during acute decompensation should include dextrose-containing IV fluids, stopping exposure to potentially toxic medications, and correction of any metabolic derangements. (Note: dextrose should be provided only in limited in quantity or not at all, as indicated by clinical status in suspected or confirmed disorders of pyruvate metabolism, if the patient is on a ketogenic diet, or the patient has had an adverse response to high glucose delivery.) IV fluid rate should be based on the clinical situation. Outpatient mitochondrial therapies should be continued when possible.

7. Lipids can be used when needed in mitochondrial patients, even in the presence of secondary fatty acid oxidation dysfunction.

8. The following medications should be avoided in patients with mitochondrial disease when possible and, if given, they should be used with caution: valproic acid; statins; metformin; high-dose acetaminophen; and selected antibiotics, including aminoglycosides, linezolid, tetracycline, azithromycin, and erythromycin.

9. Repeat neuroimaging should be considered in any mitochondrial patient with an acute change in neurologic status.

Anesthesia and surgical management ${ }^{3}$

1. Patients with mitochondrial diseases are at an increased risk of anesthesia-related complications.

2. Preoperative preparation of patients with mitochondrial disease is crucial to their perioperative outcome. Patients should minimize preoperative fasting and have glucose added to their perioperative IV fluids, unless they are on a ketogenic diet or have been demonstrated to have adverse reaction to higher glucose intake.

3. Caution must be used with volatile anesthetics because mitochondrial patients may potentially be hypersensitive.

4. Caution must be used with muscle relaxants in those mitochondrial patients with a preexisting myopathy or decreased respiratory drive.

5. Mitochondrial patients may be at a higher risk for propofol infusion syndrome and propofol use should be avoided or limited to short procedures.

6. One should consider slow titration and adjustment of volatile and parenteral anesthetics to minimize hemodynamic changes in mitochondrial patients.

7. Local anesthetics are generally well tolerated in patients with mitochondrial defect.

8. There is no clear established link between malignant hyperthermia and mitochondrial disease.

Stroke management ${ }^{3,72}$

1. Strokelike episodes in primary mitochondrial disease typically have correlating visible magnetic resonance imaging abnormalities.

2. IV arginine hydrochloride should be administered urgently in the acute setting of a strokelike episode associated with the MELAS m.3243A>G

mutation in the MTTL1 gene and considered in a stroke-like episode associated with other primary mitochondrial cytopathies as other etiologies are being excluded. Patients should be reassessed after 3 days of continuous IV therapy.

3. The use of daily oral arginine supplementation to prevent strokes should be considered in MELAS syndrome.

4. The role of monitoring plasma arginine and citrulline levels and oral citrulline supplementation in the treatment of MELAS requires further research.

a. More frequent monitoring is needed in patients with large-scale mtDNA deletions due to the high risk of heart block and either of two of the more common pathogenic mtDNA mutations (m.3243A $>\mathrm{G}$ and m.8344A $>\mathrm{G}$ ) owing to the high risk of ventricular pre-excitation.

b. Ablation should be considered in supraventricular tachycardia, Wolf-Parkinson-White or any arrhythmias potentially treatable by this technique.

c. A low threshold for pacemaker implantation is needed to prevent cardiac death. Pacemakers can be combined with implantable defibrillator if needed.
An implantable cardioverter defibrillator is needed in patients at risk of sudden death, when the left ventricle wall thickness is $>30 \mathrm{~mm}$, and in patients with ventricular tachycardia (sustained and nonsustained).

5. Cardiac magnetic resonance imaging is needed for patients with inconclusive echocardiographic images to identify structural remodeling, to quantify abnormalities more precisely prior to starting a treatment, and when invasive therapies like septal myectomy are considered. 
Table 4 Medication cautions

\begin{tabular}{|c|c|c|}
\hline Medication & Common uses & Concern in mitochondrial disease \\
\hline Acetaminophen & Analgesic, fever prevention, headaches & Chronic or frequent use may deplete glutathione and cause hepatopathy \\
\hline Aminoglycosides & Antibiotic & Hearing loss \\
\hline Antiretrovirals & HIV therapy & $\begin{array}{l}\text { Impaired mtDNA replication and worsening peripheral neuropathy, liver } \\
\text { dysfunction, or myopathy }\end{array}$ \\
\hline Botulinum toxin & Dystonia, spasticity & Worsening of weakness \\
\hline Butterbur & Headache & May contain pyrrolizidine alkaloids (oxidants) and cause hepatopathy \\
\hline Statins & Hypercholesterolemia & Worsening myopathy and elevated creatine kinase \\
\hline Valproic acid & $\begin{array}{l}\text { Epilepsy, headache, mood disorders, movement } \\
\text { disorders, tone abnormalities }\end{array}$ & $\begin{array}{l}\text { Irreversible liver failure and onset of hepatoencephalopathy, especially in } \\
\text { POLG-related disorders; worsening of seizures }\end{array}$ \\
\hline Vigabatrin & Epilepsy & $\begin{array}{l}\text { Inhibition of the mitochondrial nucleoside salvage pathway and worsening of } \\
\text { mtDNA depletion disorders }\end{array}$ \\
\hline
\end{tabular}

mtDNA, mitochondrial DNA.

With the exception of valproic acid in POLG-related disorders, these medications are not contraindicated and may be used with caution.

6. Other comorbidities should be assessed, including high blood pressure, diabetes, and dyslipidemias, and should be treated accordingly.

7. Exercise testing and/or stress echocardiography should be performed under close monitoring to assess functional capacity, response to therapy and exercise-induced dynamic left ventricular outflow tract obstruction. This test should not be performed in all patients; the potential health risks (exercise intolerance, metabolic decompensation) should be weighed against the expected benefits of such a test.

8. If physical activity is possible, the intensity of the physical activity should be prescribed by the cardiology team, according to the limitations of their cardiac function.

9. In the setting of end-stage heart failure, transplantation may be an option. Decisions to list a patient for transplant should be taken in the light of other comorbidities and the known natural history of the specific mitochondrial disease.

\section{Critical care}

Patients with metabolic diseases, including mitochondrial disease, are vulnerable to episodic decompensation and worsening of symptoms during intercurrent illness. These episodes of critical illness may be precipitated by catabolism and increased systemic energy demands during times of infection, fasting, anesthesia, and surgery. In some cases, no acute precipitating events are apparent. Prompt intervention is essential as many patients respond to timely intervention.

Initial triage stratification of critically ill mitochondrial patients should include a systemic assessment of all body systems since the disease is multisystemic and patients may develop new organ system involvement during an acute decompensation. Cardiopulmonary failure and acute cerebral events have been described as the leading cause of mortality in adult mitochondrial patients. ${ }^{15}$ Patients with mitochondrial disease are more likely to have underlying myopathy, immobility, dysphagia, chronic intestinal pseudo-obstruction (CIPO), cardiomyopathy, and cardiac arrhythmias including Wolf-Parkinson-White syndrome-all increasing their risk of cardiorespiratory compromise during illness or medical stress. Renal dysfunction with alterations in fluid and electrolyte homeostasis and impairment of acid-base balance is a potential cause of morbidity and mortality. Tubulointerstitial disease is the most common acute renal manifestation. Focal segmental glomerulosclerosis, steroid resistant nephrotic syndrome, and end-stage renal disease during acute illness have also been described. ${ }^{16}$ Endocrinopathies can manifest during critical illness in mitochondrial patients. Diabetes mellitus due to insulin insufficiency secondary to islet cell dysfunction or insulin resistance, ${ }^{17-19}$ primary adrenal insufficiency, thyroid dysfunction, hypoparathyroidism, and hypogonadism are also seen. ${ }^{20,21}$ Patients with mitochondrial diseases may be chronically malnourished with deficiencies of both macro- and micronutrients. Decreases in nutritional intake due to poor oral or enteral tolerance, in the setting of gastrointestinal (GI) dysmotility and paralytic ileus may further compromise nutrition in ill states. A recent study has shown a higher burden of strokes following acute worsening of CIPO in MELAS patients. ${ }^{22}$

\section{Recommendations}

The recommendations below are in addition to routine intensive-care management that might be undertaken for any critically ill patient. They are in addition to routine illness consensus guidelines published previously ${ }^{3}$ and summarized in Table 3.

1. Patients should be provided a summary document by their mitochondrial disease care center of clinical symptoms, organ specific symptoms, baseline cardiorespiratory function, special nutritional needs, medications, allergies, and contraindicated therapies. The 
document should include anticipatory guidelines for management during critical illness. The document must be updated at each follow-up visit and be readily available for review in an emergency.

2. Critically ill mitochondrial disease patients must be carefully triaged at presentation and referred to a center equipped to manage multisystem dysfunction when necessary.

3. Ventilatory assessment should include evaluation for aspiration, atelectasis, and infection, and account for underlying issues of abnormal tone, as well as nerve and muscle disease.

4. An echocardiogram should be obtained in instances of hemodynamic dysfunction.

5. In patients with pressor-refractory hypotension, empiric administration of stress-dose steroids should be considered; blood levels of adrenocorticotropic hormone and cortisol can be collected immediately prior to facilitate the diagnostic evaluation but their collection should not delay therapy.

6. Electrolyte assessment should include sodium, potassium, calcium, magnesium, and phosphate. Recurrent or persistent electrolyte imbalances should prompt an evaluation of renal tubular and adrenal function.

7. Patients may acutely develop elevations in lactate or pyruvate resulting in decompensated acidosis and should be monitored closely particularly during introduction of feeds or weaning from respiratory support.

8. Thyroid and adrenal function should be assessed in patients at times of critical illness and reassessed during a prolonged intensive care unit stay. Hypo- and hyperglycemia can occur and regular blood glucose monitoring is needed.

9. Any change in baseline neurological exam warrants consideration for repeat neuroimaging.

10. MELAS patients with pseudo-obstruction should receive closer neurologic follow-up as the symptom may represent a harbinger of stroke.

11. Nutritional support must be provided with enteral feeds if tolerated or with peripheral or central parenteral nutrition that is calculated with the help of a trained dietitian. Nutritional support should be started as soon as it is medically safe to do so (preferably within 48 hours of admission) to avoid a prolonged catabolic state that may lead to further metabolic decompensation.

12. Hypoglycemia, hypoalbuminemia, or coagulopathy must be excluded if liver dysfunction is suspected. Importantly, hepatocerebral involvement is common in infantile mitochondrial disease; the use of valproate for seizure control must be avoided in these patients as fulminant hepatic failure has been associated with this medication.

13. In patients with marrow failure, transfusions with packed red cells or platelets may be necessary. Granulocyte colony-stimulating factor may be required in the setting of severe neutropenia and/or infections. Additional studies and interventions may require the services of a hematologist.

14. Supplements and cofactor therapy should be continued when possible.

\section{Endocrinology}

Mitochondrial disease patients are at risk for a variety of endocrine diseases. Recent reviews on the topic are available. $^{20,21}$ Diabetes mellitus has long been a well-recognized potential endocrine consequence of mitochondrial disease. ${ }^{23}$ Clinical presentations suggestive of either "typical" type I or II diabetes mellitus can both be caused by primary mitochondrial impairment (mitochondrial disease-associated diabetes mellitus). Mitochondrial-related diabetes mellitus may present insidiously, clinically similar to more "typical" age- and weight-related type 2 diabetes mellitus with both insulin resistance and inadequate pancreatic beta-cell insulin production. Children may present with more features of insulin deficiency. Diabetes mellitus is one of the most common findings in patients with the m.3243A $>$ G mutation, typically manifesting as maternally inherited diabetes and deafness. ${ }^{17-19}$

Both hypothyroidism and, to a far lesser extent, hyperthyroidism have been reported in patients with primary mitochondrial diseases and may coexist with other endocrinopathies. ${ }^{20,21}$ Short stature is common. There may be failure of sufficient growth hormone $(\mathrm{GH})$ production and some children may benefit from GH supplementation. ${ }^{24}$ Hypoparathyroidism has been reported in some subtypes of mitochondrial diseases, most notably KSS. Concurrent renal tubulopathy in KSS can make both diagnosis and management more challenging. ${ }^{25}$ Individuals with mitochondrial disorders may also be at risk for poor bone health, particularly if they are chronically ill, malnourished, and immobile. Certain medications, such as antiepileptic drugs, may compound the issue.

Adrenal insufficiency can also occur in primary mitochondrial diseases and consists mostly of either primary adrenocortical insufficiency, due to diminished cortisol secretion (Addison disease), or to hyperaldosteronism. Most described cases have been associated with Pearson or KSS, MELAS, and $P O L G$-related disease. ${ }^{20,21}$ Primary hypoglycemia is not a common occurrence in mitochondrial disease and is usually related to hepatic impairment. The presence of hypoglycemia should prompt screening for reversible causes (e.g., adrenal insufficiency, GH deficiency, hyperinsulinism). Primary or hypergonadotropic and hypogonadotropic hypogonadism have been observed, particularly in POLG-related disease ${ }^{26}$ and myopathies presenting with progressive external ophthalmoplegia. ${ }^{21,27}$

\section{Recommendations}

1. An annual hemoglobin Alc (HgbAlc), thyroid-stimulating hormone, free thyroxine level (FT4), vitamin D, and screening for hypoparathyroidism (serum calcium, 
magnesium, phosphate, parathyroid hormone, vitamin D (25-OHD and 1,25-OHD); urine: creatinine, calcium, and phosphate) can be considered in individuals with mitochondrial diseases.

a. In those with mtDNA deletions, which are more strongly associated with secondary endocrinopathies, annual screening is recommended.

2. Biguanides such as metformin may need to be avoided or used with caution in mitochondrial disease because they may exacerbate lactic acidosis.

3. Multiagent therapy may be needed, including insulin, for patients with mitochondrial diabetes as they may have both insulin resistance and insulin deficiency.

4. Growth and maturation in children with mitochondrial disorders should be measured consistently at routine well-child primary care physician visits. If short stature and/or suboptimal growth and weight gain occur, a comprehensive evaluation should be undertaken, guided by the history and physical examination, and with attention to treatable causes, including GH deficiency.

a. Nutritional status should be assessed in all children with decreased linear growth velocity.

b. In patients with $\mathrm{GH}$ deficiency, treatment with $\mathrm{GH}$ should be considered.

c. Atypical patterns of sexual maturation should prompt investigation for hypogonadism.

5. Adrenal insufficiency should be considered in mitochondrial patients with new onset of worsening fatigue or symptoms typically associated with adrenal insufficiency.

6. Optimization of calcium and vitamin D status and attention toward injury/fall prevention are reasonable steps to prevent occult and/or atraumatic fracture. Bone density testing should be considered in patients with encephalopathy or immobility to assess for fracture risks.

\section{Gastroenterology}

Mitochondrial diseases often feature GI system involvement including intestinal dysmotility, constipation, poor weight gain, obesity, swallowing dysfunction, and pancreatic and hepatic dysfunction. A comprehensive review has recently been published. ${ }^{28}$

GI dysmotility (including CIPO in severe cases) is a relatively common feature of many mitochondrial diseases and may present with early satiety, weight loss, nausea, constipation, or overflow incontinence/diarrhea. Constipation can also present without dysmotility due to underlying myopathy, neuropathy, medication effects, dietary alterations, decreased fluid intake, and decrease in patient mobility. CIPO is characterized by more than 6 months of severe symptoms of intestinal obstruction such as abdominal distention and pain, nausea, and vomiting with clinical/radiographic evidence of dilated bowel in the absence of mechanical obstruc- tion. $^{29}$ CIPO may be caused by neurologic or myopathic defects or secondary to medications. ${ }^{30,31}$ A recent study has shown a potential higher burden of strokes following acute worsening of CIPO in MELAS patients. ${ }^{22}$

Any combination of dysmotility, constipation, and/or dysphagia can lead to chronic nausea, vomiting, poor appetite, and exacerbate poor weight gain and ongoing malnutrition seen as a metabolic consequence of mitochondrial disease. Obesity is a less common feature of mitochondrial disease. Patients with primary mitochondrial disease are also at risk of aspiration pneumonia, due to oropharyngeal weakness or dyscoordination, compounded by gastroesophageal reflux disease. The use of a gastrostomy or gastrojejunal tube and consideration for fundoplication can improve nutritional status and decrease aspiration and gastroesophageal reflux diseaserelated risks.

Exocrine pancreatic insufficiency can occur, especially in Pearson syndrome, KSS, and patients with the m.3243A > G mutation. ${ }^{32,33}$

Patients with mitochondrial disease are at risk for liver dysfunction and liver failure. While typically presenting with multisystem manifestations, isolated liver disease is most frequently caused by defects of mtDNA maintenance such as mtDNA deletion and depletion, especially in those with POLG, DGUOK, and MPV17 mutations. ${ }^{28}$ Patients with mutations in $P O L G$ are at a higher risk of developing valproate-induced liver failure. Antipyretic therapy and induction of anabolism including glucose and parenteral lipids may ameliorate the course of liver crises in certain patients as has been noted in NBAS deficiency. ${ }^{34}$

Liver transplantation may be indicated in some cases and has been performed in patients with mitochondrial disease. Survival after liver transplantation in some mitochondrial patients approaches that of transplanted patients without mitochondrial disease, although some patients may fare worse (POLG-related disease). ${ }^{35-37}$

\section{Recommendations}

1. Mitochondrial patients should be screened for symptoms of upper and lower tract dysmotility including nausea, early satiety, and constipation. Persistent symptoms or physical examination or radiologic findings of impacted stool should prompt referral.

2. Diarrhea is not a common symptom in mitochondrial disease (outside of overflow incontinence from constipation) and should lead to an investigation as to the etiology including pancreatic insufficiency.

3. Acute pseudo-obstructive presentations should be managed aggressively. Water soluble contrast enemas may relieve obstruction and allow for exclusion of alternate etiologies such as volvulus. Patients should be nil-bymouth at these times; Intravenous (IV) hydration with dextrose and electrolytes should be provided. Onset of pseudo-obstruction in MELAS patients may be a harbinger of neurologic decline including new strokes and patients should be closely monitored at these times. 
4. Any cases with suspected aspiration or bulbar dysfunction should be evaluated with a swallow study and advise on feeding strategies by a speech therapist.

5. Decisions regarding gastrostomy or jejunostomy tube insertion should be made in close consultation with a gastroenterologist and, in some cases, palliative care.

6. All patients with a mitochondrial disease diagnosis should undergo surveillance for malnutrition, including measurement of weight and weight for height, no less often than every 6 months.

a. Mitochondrial patients with growth failure should be screened for pancreatic insufficiency.

b. All patients identified on anthropometric examination as at risk for malnutrition should be seen by a dietitian with experience in the management of patients with neuromuscular or mitochondrial disease.

c. Standard markers of nutritional status, vitamin levels, and trace elements can help guide therapy in addition to monitoring body mass.

d. Diet/exercise programs that promote lean muscle mass should be promoted over simply increasing body mass index.

e. If obesity is present, careful management is indicated to ensure weight loss that may improve functional ability without exacerbating other underlying symptoms.

7. If patients have symptoms or laboratory values indicative of exocrine pancreatic insufficiency, referral to a gastroenterologist is indicated.

8. Patients with mitochondrial disease should have an annual transaminase level assessed; synthetic liver function should be evaluated if transaminases are elevated or if the patient is symptomatic. Elevated liver enzymes or laboratory markers of hepatic synthetic deficiency should prompt referral to a hepatologist.

9. In the setting of acute or acute on chronic liver failure, liver transplantation may be an option. Decisions to list a patient for transplant should be taken in the light of other comorbidities and the known natural history of the specific mitochondrial disease. Additional caution regarding liver transplantation may be needed in patients with $P O L G$ mutations owing to the potential of increased morbidity and mortality.

\section{Hematology}

Hematologic disease is not a common occurrence in mitochondrial patients although mild anemia is observed relatively frequently. Sideroblastic anemia is a known classical feature of several rare mitochondrial diseases, including Pearson syndrome. Other types of anemia, leukopenia, neutropenia, thrombocytopenia, and pancytopenia have also been described although these are not seen frequently. A review on the topic is available. ${ }^{38}$ Anemia and/or iron deficiency may also occur due to feeding or GI issues potentially compounding symptoms of fatigue.

\section{Recommendations}

1. A complete blood count with differential should be considered annually in patients with primary mitochondrial disease. Patients with mitochondrial diseases at higher risk of anemia or bone marrow suppression (such as Pearson syndrome) should have complete blood count checked more frequently, with the interval based on the patient's clinical course.

2. Iron studies should be considered in patients at risk of nutritional deficits and/or concomitant symptoms of fatigue.

\section{Immunology}

The immune system and its interplay with mitochondria remains poorly understood, ${ }^{39}$ although in vitro studies have repeatedly demonstrated that mitochondria are crucial for proper function of both the cellular and humoral immune systems. ${ }^{40-42}$ Certain conditions such as pancytopenia or neutropenia have been well described in disorders such as Barth and Pearson syndromes. ${ }^{43,44}$ The most comprehensive study investigating immune function and/or risk of infections in patients with mitochondrial disease included a large cohort without genetically confirmed disease. ${ }^{45}$ Patients in this cohort seemed to be at a higher risk of recurrent infections and sepsis and some showed abnormalities on immune testing including hypogammaglobulinemia, low vaccine titers, and low switched memory B-cell compartment. ${ }^{45}$

Specific studies have not investigated the safety of vaccine administration in children with mitochondrial disease, although reviews of patient outcomes in other inherited metabolic disorders show that vaccines are generally safe. ${ }^{46}$ The MMS recommends vaccination for all patients with mitochondrial disease since risk of their mitochondrial disease worsening from life-threatening infections is likely to be greater than the theoretical risk of decompensation following vaccination. ${ }^{47}$

\section{Recommendations}

1. Immune function should be evaluated early in any mitochondrial disease patient experiencing recurrent or severe infections. ${ }^{*}$ Investigation should include quantitative immunoglobulin levels, vaccine-specific IgG titers, and lymphocyte subset levels ( $T$ cell, B cell, switched memory B-cell compartment, natural killer cells). Immune evaluation and management should be conducted with the assistance of an immunologist familiar with mitochondrial disease.

${ }^{*}$ Recurrent or severe infections are defined as those that are complicated, in multiple locations, resistant to treatment, caused by unusual organisms, or consisting of $>10$ upper respiratory infections a year. 
2. Immune testing may be useful to identify patients that may benefit from prophylactic treatment strategies including antibiotic prophylaxis, immunoglobulin replacement therapy, and granulocyte colony-stimulating factor.

3. Patients with mitochondrial diseases should be offered age-appropriate vaccination including the influenza vaccine as well as other relevant vaccines when there is an underlying pulmonary pathology (i.e., pneumococcal vaccine).

\section{Nephrology}

Kidneys contain a high density of mitochondria, particularly in the cortical tubules, and all sections of the nephron may be affected by mitochondrial disease. In general, renal tubular dysfunction is more frequently seen in childhoodonset mitochondrial disease, whilst glomerular disease is more frequent in adults, although exceptions do occur. The prevalence of renal dysfunction in mitochondrial disease is not known, since the published literature consists largely of single case reports and small case series. Recent reviews on the topic are available. ${ }^{16,48}$

Mild tubular dysfunction is one of the most common findings in a variety of mitochondrial disease patients, ${ }^{49}$ especially those with mtDNA deletion disorders. Fanconitype proximal tubulopathy with severe electrolyte losses, tubulointerstitial nephritis, Bartter-like syndrome, renal tubular acidosis, and a Gitelman-like hypomagnesemia have all been described and are seen in a range of mitochondrial diseases. ${ }^{16}$

Glomerular dysfunction, including steroid-resistant nephrotic syndrome, which may progress to focal segmental glomerulosclerosis, may also occur in mitochondrial disease and has been associated with a variety of mtDNA and nuclear DNA defects. The common m.3243A > G pathogenic variant, often associated with MELAS, has most commonly been associated with focal segmental glomerulosclerosis. ${ }^{16}$ Chronic tubulointerstitial nephritis has also been noted in some.

Patients have at times needed dialysis and renal transplantation. Most reported cases with mitochondrial disease undergoing renal transplantation have fared well and survival in mitochondrial patients matches national averages. ${ }^{36}$

\section{Recommendations}

1. All patients with suspected mitochondrial disease should be screened for renal dysfunction at presentation, including blood urea, creatinine, sodium, potassium, calcium, magnesium, phosphate, and urinary albumin/creatinine ratios.

2. Patients with significant renal dysfunction should be referred to a nephrologist, preferably with experience of mitochondrial disease, who can guide measurement of glomerular filtration rate and need for renal replacement therapy.

3. Electrolyte replacement-large volumes of potassium, calcium, magnesium, and/or phosphate-may be needed, and consideration should be given to the early placement of a gastrostomy tube if the patient struggles to take these supplements orally.

4. Dialysis for end-stage renal disease should be considered palliative, and patients receiving dialysis should ideally also be considered for renal transplantation.

5. Renal transplantation should be offered after careful multidisciplinary review and discussion with the affected family and consideration of the full clinical picture.

\section{Neurology}

\section{Developmental disability}

Since mitochondrial diseases frequently cause neurologic impairment as well as vision and/or hearing impairment, patients with mitochondrial diseases are at risk for global developmental disability and intellectual disability (ID). Mitochondrial diseases can cause a variety of developmental disabilities in isolation or in various combinations, including ID, autism, cerebral palsy, or isolated learning disabilities. Attention deficit disorder with or without hyperactivity can be seen. Concomitant or isolated language or motor disability can also be present. Mitochondrial diseases due to point mutations in nuclear DNA or mtDNA, large-scale mtDNA deletions, or mtDNA depletion can all present with isolated or global developmental delay and/or ID. For a given disease within the same family, the severity of intellectual and developmental delays or disabilities can be quite broad. One affected family member may have preserved cognition while another affected individual in the same family may have significant ID.

Many mitochondrial diseases can also cause loss of developmental and cognitive skills, referred to a "neuroregression" or "psychomotor regression." Neuroregression is a semiologically distinct entity from global developmental disability/ ID. Regression can occur at any age and frequently leads to life-limiting neurologic impairment. Such regression warrants a thorough and expedited evaluation for a treatable etiology.

\section{Recommendations.}

1. As patients with mitochondrial disease are at risk of developmental disabilities, every patient deserves a formal neurodevelopmental screen appropriate for age.

2. Baseline neuropsychological testing should be considered for mitochondrial patients with CNS disease. Testing should be repeated for cognitive, behavioral, or personality change and to allow for optimal tracking of their function and development of personalized care plans.

3. If developmental disability/ID is confirmed, then one should consider referrals to a developmental specialist and appropriate therapist, and for community and school support services. Anticipatory guidance should be provided to parents, siblings, and family.

4. Concomitant symptoms such as autism, attention deficit disorder with or without hyperactivity, and psychiatric disease should be addressed and treated. 
5. Neurodevelopmental and cognitive status should be closely monitored at each follow-up since mitochondrial patients can be at risk of neurologic regression.

6. Developmental regression at any age warrants a thorough evaluation for a potential treatable condition as well as an assessment for disease progression; testing may need to include repeat neuroimaging and an electroencephalogram.

\section{Epilepsy}

The prevalence of epilepsy in mitochondrial disease is not accurately known although seizures may be a presenting or late feature. ${ }^{50}$ Seizures are reported to occur in $35-60 \%$ of infants, children, and adolescents with biochemically or genetically confirmed disease. ${ }^{50-53}$ The prevalence in adults is potentially lower with one study reporting a general prevalence of $23 \%$; certain genotypes such as the m.3243A $>$ G and m.8344A $>G$ mutations may have a higher prevalence. ${ }^{54}$ Focal and generalized seizures, epilepsia partialis continua, myoclonus, and infantile spasms have all been noted. Patients may also have progressive myoclonic epilepsy or recurrent status epilepticus. Electroencephalogram findings vary. ${ }^{55}$

Treatment of epilepsy is similar to non-mitochondrial disease patients with seizures. However, valproic acid should be avoided, especially in patients with POLG mutations, due to the risk of fulminant hepatic failure and worsening of neurologic symptoms, including seizures. Vigabatrin may also need to be avoided in patients with mtDNA depletion because it inhibits the conversion of deoxyribonucleoside diphosphate to deoxyribonucleoside triphosphate in the mitochondrial nucleoside salvage pathway and may worsen mtDNA depletion. ${ }^{56}$ Topiramate may worsen acidosis. ${ }^{57}$ Epilepsy may be refractory to treatment. No specific combination of medications has been shown to be more beneficial.

\section{Recommendations.}

1. In mitochondrial patients with recurrent stereotypical spells, episodes of behavior arrest or alterations of state from baseline there should be a low threshold to obtain electroencephalogram monitoring, especially in those with underlying cognitive disability or dementia.

2. Avoid the use of valproic acid, especially in patients with $P O L G$-related disease. Vigabatrin may need to be avoided in patients with mtDNA depletion syndromes. Topiramate may worsen acidosis.

3. Epilepsia partialis continua, when present, should raise the concern of a metabolic stroke.

\section{Headaches}

Headaches are seen more frequently in several mitochondrial diseases than in the general population and can represent a manifesting feature of select mitochondrial diseases (MELAS, myoclonic epilepsy with ragged red fibers, Alpers-Huttenlocher, and carriers of m.3243A > G). ${ }^{58-60}$

Severe headaches in MELAS patients have been associated with strokelike episodes and seizures. ${ }^{58}$ There are no studies looking at the treatment of headache in patients with mitochondrial disease. Most typical migraine therapies, including triptans, can be used. There are some medications that may need to be avoided or used with caution; butterbur products contain pyrrolizidine alkaloids, which are strong oxidants, and theoretically may induce liver damage. ${ }^{61}$ Longterm acetaminophen use can reduce hepatic reduced glutathione and potentiate oxidative hepatic damage. Topiramate may worsen acidosis. ${ }^{57}$ Valproate should be avoided due to the risk of liver failure, especially in POLG-related diseases.

\section{Recommendations.}

1. Sudden-onset or worsening severity of migraines in mitochondrial disease may represent underlying strokes or seizures, requiring further evaluation for these conditions at that time.

2. Treatment for migraines in mitochondrial disease is similar to that in patients without mitochondrial disease.

a. Caution may be needed with use of topiramate, butterbur, and chronic acetaminophen for headache management in mitochondrial disease patients. Valproic acid should be avoided; it is contraindicated in patients with $P O L G$ mutations.

\section{Movement disorders and altered tone}

Patients with primary mitochondrial disease are vulnerable to injury to the basal ganglia, cerebellum, cortex, or corticospinal tracts, and are thus at risk of developing movement disorders and abnormalities of tone. Patients can have a mixed movement and tone disorder that includes hyper- and hypokinetic or cerebellar types of movements, hypotonia, spasticity, rigidity, and dystonia. ${ }^{62}$ Myoclonus, ataxia, gait disturbance, Parkinsonism, and rigidity have also been noted. Patients with POLG mutations or mtDNA depletion syndromes are at a higher risk for Parkinsonian symptoms. ${ }^{59,62-66}$

\section{Recommendations.}

1. Routine neurologic assessment for disorders of movement and tone is needed.

2. Sudden onset of a new disorder of movement or tone should lead to an evaluation of disease worsening and potential secondary causes; neuroimaging, electrophysiological testing, and baseline laboratory investigations are recommended. Assays of cerebrospinal fluid neurotransmitter and folate levels should be considered and treatable causes should be excluded.

3. Management of movement and tone disorders in mitochondrial disease is similar to that in patients with movement disorders of other causes. Medical, procedural, and surgical treatments should be considered. Treatment can improve quality of life.

a. Caution should be used with medications that alter tone as they can selectively worsen cognitive status, decrease muscle strength and secondarily respiratory effort, and impact GI motility and urinary function. 
b. Valproic acid should be avoided as a treatment, especially in $P O L G$ disease.

c. Botulinum toxin should be used with caution in view of potential systemic effects.

4. Physical medicine and therapy assessments are recommended to maximize mobility, prevent contractures, and alleviate discomfort and pain.

5. Deep brain stimulation should be considered when appropriate for treatment of mitochondrial movement disorders, rigidity, and dystonia, considering the patient's long-term prognosis and level of morbidity.

\section{Myopathy}

Myopathy is a common manifestation of some mitochondrial diseases and may include ophthalmoplegia and ptosis. Earlyonset myopathy may present with profound hypotonia in infancy. Patients are at risk of associated dysphagia, respiratory insufficiency, cardiomyopathy, exercise intolerance, myalgia, fatigue, and infrequently rhabdomyolysis. Mitochondrial myopathies do not typically lead to marked baseline elevations in creatine kinase (CK) (with the exception of the myopathic form of TK2-related mitochondrial disease).

\section{Recommendations.}

1. An initial evaluation of muscle function is routinely needed including assessment of strength, CK measurement, and consideration of an electromyogram.

2. Annual CK levels should be considered in patients with a myopathy, especially with symptom worsening or a decline in function.

3. Secondary causes of a myopathy should be considered and assessed for, especially if CK levels are above 1,000 (other than in TK2-related disease) and include at least a complete blood count, thyroid-stimulating hormone, and toxicology screen when appropriate.

4. Management for recurrent rhabdomyolysis and myoglobinuria, at times triggered by exercise or illness, should follow general treatment recommendations; acylcarnitine and urine organic acid analysis may be needed to exclude fatty acid oxidation defects in new cases.

5. Exercise may benefit patients with mitochondrial myopathies (see prior exercise recommendations). ${ }^{3}$

6. Agents such as statins, corticosteroids, metformin, and antiretrovirals should be used with caution since they may exacerbate the underlying myopathy.

7. Riboflavin should be considered for myopathy associated with ACAD9 deficiency; a combination of coenzyme $\mathrm{Q}_{10}$ and riboflavin should be considered for $E T F D H$-related myopathy.

\section{Neuropathy}

Mitochondrial diseases can lead to primary neuropathy. Large and small fiber, axonal, and demyelinating neuropathies have all been described, often presenting subclinically. ${ }^{67-71}$ Peripheral neuropathy may be more common in POLG-,
TYMP-, MPV17-, and SURF1-related mitochondrial diseases and in patients with the m.8993T $>$ G and m.8993T $>C$ mtDNA mutations. Neuropathy can also occur secondarily as a complication of mitochondrial diabetes, renal insufficiency, or side effects from treatments.

\section{Recommendations.}

1. Mitochondrial disease patients should be screened for peripheral neuropathy when clinical symptoms/signs suggestive of peripheral nerve dysfunction are present. A comprehensive electromyogram and nerve conduction velocity study should be considered and should include both upper and lower extremities with evaluation of both sensory and motor nerves.

2. Screening for treatable causes of peripheral neuropathies should be obtained, especially when the findings are atypical for the underlying mitochondrial disease. As diabetes may be more common in certain mitochondrial diseases including MELAS and maternally inherited diabetes and deafness, optimal glycemic control may help a sensory polyneuropathy.

3. Treatment of mitochondrial neuropathies is symptomatic and follows guidelines established for the care of nonmitochondrial neuropathies. Caution and close monitoring is needed if medications causing mitochondrial toxicity are used. Valproate should be avoided as a potential treatment, especially in patients with $P O L G$ mutations.

\section{Stroke}

The data for the management of mitochondrial stroke were previously reviewed with recommendations published by the MMS. ${ }^{3,72}$ These recommendations are summarized in Table 3.

\section{Ophthalmology}

The ophthalmologic involvement in mitochondrial disease is diverse and may be the dominant feature, such as ophthalmoplegia and ptosis in various chronic progressive external ophthalmoplegia conditions; or specific for a syndrome, such as optic nerve disease in Leber hereditary optic neuropathy (LHON). Alternatively, the ophthalmologic manifestations may be nonspecific, such as cataracts, retinal disease, nystagmus, strabismus, or decreased visual acuity. Involvement of the iris and ciliary body, choroid, and uvea are rare in mitochondrial disease, compared to involvement of the lens (refractive errors and cataracts), retina (pigmentary retinopathy or macular degeneration), optic nerve (optic nerve atrophy), extraocular muscles (ophthalmoplegia), and levator palpebrae (ptosis). The reported frequency of ophthalmologic manifestations in mitochondrial disease is variable ranging from $35 \%$ to $81 \%{ }^{73-75}$ and has been described in multiple types of mitochondrial diseases.

LHON is mentioned separately owing to its relatively high prevalence and several unique features of the disease. The disorder is a common mitochondrial optic neuropathy leading to an acute onset of painless, bilateral central vision 
loss, often developing in the young adult years. Heavy alcohol and moderate nicotine/tobacco exposure increase the risk of symptoms developing and avoidance of these environmental triggers is recommended. Some patients with LHON appear to develop addition neurologic symptoms or cardiac conduction defects. ${ }^{76}$ Idebenone is a potential treatment. ${ }^{77,78}$

\section{Recommendations}

1. A thorough clinical evaluation for eye disease should include visual acuity, visual fields, eye motility exam, documentation of ptosis, and fundoscopy regardless of reported symptoms.

2. Patients should be referred to a neuro-ophthalmologist for detection of visual, retinal, macular, and optic nerve changes at the time of diagnosis, supported by optical coherence tomography and electroretinography. If symptomatic, an assessment of intraocular pressure is indicated. Annual ophthalmology exams should be conducted thereafter.

3. Patients with visual impairment should be referred to a low vision specialist.

4. Surgery may be beneficial for strabismus or cataracts. Surgery for ptosis may be needed and should be conducted by a surgeon experienced in treating patients with mitochondrial disease.

5. Lubrication of the eyes may be needed due to inappropriate eyelid spread of tears in patients with ptosis or after ptosis repair.

6. Heavy alcohol use and smoking should be avoided among LHON carriers as these are strongly associated with increased risk of visual loss.

7. LHON patients should receive periodic neurologic evaluations and annual ECG.

\section{Orthopedics}

Orthopedic complications occur in mitochondrial disease due to a variety of causes including underlying myopathy, neuropathy, abnormalities of tone, strokes, and basal ganglia or cerebellar disease. Orthopedic interventions may be necessary for contractures, scoliosis, hip dislocation, and limb deformities. Regular orthopedic examination of patients with mitochondrial diseases may be indicated. Treatment is symptomatic and peer reviewed data is lacking for the efficacy of specific orthopedic interventions in mitochondrial diseases. Recommendations are based on those made for similar issues for patients with nonmitochondrial diseases.

\section{Recommendations}

1. Patients should be examined annually for orthopedic complications including scoliosis, contractures, dislocations, and limb deformities, especially if the patient has underlying abnormalities in tone, muscle strength, or neurologic functioning.

2. Evaluation and care by a rehabilitation medicine specialist and therapy are used to maintain safety, mobility, and an active lifestyle.
3. Inpatient rehabilitation may improve function in some patients and should be considered when needed.

4. Orthopedic interventions, both operative and nonoperative, for scoliosis, dislocations, and limb deformities may be beneficial to selected patients. As with all procedures, life expectancy should be weighed against risks of discomfort and recovery time when considering an orthopedic procedure.

\section{Pregnancy}

There are mostly only single case reports published on the clinical course of pregnancy in mitochondrial disease patients. As a time of extreme physiologic change, pregnancy theoretically represents a period of potential risk in several areas for the pregnant woman with mitochondrial disease and her fetus. Mitochondrial patients are at risk of developing or exacerbating comorbid systemic symptoms, increasing the risk of complications during pregnancy.

Several prior case reports of women with MELAS or carriers of MELAS-related mtDNA mutations showed a risk of premature rupture of membranes, preterm labor, gestational diabetes, preeclampsia, worsening acidosis, myopathy, neuropathy, status epilepticus, and stroke. ${ }^{79-83} \mathrm{~A}$ recent retrospective analysis ${ }^{84}$ showed that obstetric complications did occur more frequently in carriers of the common m.3243A > G mutation associated with MELAS. Of 96 pregnancies reviewed, $25 \%$ had a preterm delivery $(5.5 \%$ extremely premature), $12 \%$ had preeclampsia and $11 \%$ had gestational diabetes. Thirteen percent of the children born had a birth weight below the fifth percentile. Four fetal deaths were reported.

Pregnancy in mitochondrial disease also elicits the concern of transmission of a genetic disorder. Appropriate preconception genetic counseling and discussion of options of prenatal testing are needed. A fetus affected by mitochondrial disease may also be at higher risk for prenatal morbidity. Finally, premature ovarian failure is a feature of several mitochondrial disorders and affected women should be referred for assisted reproductive technologies if they wish to have children. ${ }^{21,85}$

\section{Recommendations}

1. Pregnant women with mitochondrial disease require closer monitoring by their specialists.

2. Pregnancies should ideally be managed by an obstetrician with expertise in the management of high-risk pregnancies since affected women may be at higher risk for preterm labor and preeclampsia.

3. Women with mitochondrial disease should be counseled about a potential increased risk of gestational diabetes; glucose tolerance testing should be obtained early and later in the pregnancy.

4. Patients should be counseled about continuation or dose changes of current medications (e.g., antiepileptics, antihypertensives). 
5. Women with or at risk of mitochondrial disease should receive preconception genetic counseling and be offered prenatal testing where this is feasible.

6. Closer fetal monitoring may be required for a prenatal onset of symptoms when there is a concern of genetic transmission of a mitochondrial disorder (especially in cases of mtDNA-mediated or autosomal-dominant disease).

\section{Psychiatry}

There appears to be a potential higher prevalence of psychiatric disorders in individuals with mitochondrial disease; ${ }^{86,87}$ however, all but one study used healthy controls as a reference group. Thus, although it is important to screen for psychiatric symptoms in individuals with mitochondrial disease, more research is needed to understand whether psychiatric symptoms are specifically related to mitochondrial disease or to other factors that are common in chronic disease conditions.

Mood disorders, especially major depression, appear to be the most prevalent psychiatric disorder reported across studies. The prevalence of anxiety disorders varies and psychotic disorders are less common. ${ }^{86-88}$

\section{Recommendation}

1. A standardized screening tool for depression and anxiety symptoms should be routinely used.

\section{Pulmonology}

The lungs are not usually directly involved in the clinical manifestations of primary mitochondrial disease; however, neuromuscular and CNS involvement may affect the lungs and breathing in patients with primary mitochondrial disease. Patients with mitochondrial disease can have either central brain stem dysfunction and/or a myopathy, with or without dysphagia. Respiratory insufficiency, aspiration, and infection may also occur. Respiratory symptoms may include noisy breathing, hoarseness, stridor, congestion, cough, abnormal breathing, snoring, sleep disturbances, daytime hypersomnolence, and exercise intolerance. Case reports have outlined worsening following respiratory tract infections. ${ }^{89,90}$ Respiratory hypoventilation may also occur. ${ }^{91}$ Pulmonary hypertension has been described. ${ }^{85}$ Anesthesia may worsen respiratory symptoms and precipitate respiratory failure. Primary cardiomyopathy may lead to congestive heart failure and associated pulmonary edema, especially in the setting of respiratory muscle weakness. Polysomnography may show findings of central apnea, obstructive sleep apnea, and decreased ventilatory drive in response to hypoxia and/or hypercapnia. ${ }^{92}$

Children with more severe diseases such as Leigh syndrome tend to have more severe, early, and chronic respiratory complications compared to later-onset mitochondrial diseases where respiratory problems may only arise during acute decompensations.

Hypersialorrhea is common in patients who have CNS involvement affecting oropharyngeal strength or coordination, but it can also be caused by gastroesophageal reflux disease, constipation, and side effects of medicines. Treatment may be necessary to prevent aspiration pneumonia, improve hygiene, and reduce social stigma.

\section{Recommendations}

1. Baseline evaluation of respiratory status to assess for muscle weakness and other cardiopulmonary comorbidities. The history should guide screening for additional comorbidities including obstructive sleep apnea, bulbar weakness, risks for aspiration, gastroesophageal reflux, asthma, and chronic obstructive lung disease.

2. Initial and follow-up evaluation should include routine pulse oximetry with vital signs.

3. Polysomnograms should be considered at baseline in every patient at diagnosis to assess for sleep disturbances, central or obstructive apnea, and nocturnal hypoventilation.

4. Patients even with mild abnormalities should be referred to a pulmonary specialist for follow-up and management.

a. Spirometry in both the supine and upright positions should be considered; if appropriate seal with a mouthpiece cannot be achieved, a nasal clip or a face mask needs to be used.

b. Additional testing may be required at the discretion of a pulmonologist familiar with neuromuscular diseases that can affect the pulmonary system.

c. If initial tests are normal, repeat testing should be deferred until new symptoms arise or if there is a suspicion of disease deterioration.

d. For patients with a well-characterized respiratory involvement, testing should be repeated periodically to follow progress and predict the pulmonary function over time to guide management.

5. Neuromuscular weakness predisposes to respiratory issues in the perioperative period due to poor airway tone, clearance of secretions, and chronic aspiration.

a. Prior to surgery, pulmonary assessment should be performed, with pulse oximetry as a minimum. If $\mathrm{SpO}_{2}$ is $<95 \%$ a blood gas should be obtained to assess carbon dioxide levels.

b. For patients with neuromuscular weakness, preoperative use of noninvasive positivepressure ventilation should be considered, especially if there is a weak cough, recurrent pneumonia, or low maximum expiratory pressure.

c. Postoperatively, those with neuromuscular weakness can be extubated to noninvasive positivepressure ventilation to prevent prolonged intubation, with weaning as tolerated since recovery may be prolonged.

d. Postoperative atelectasis may require aggressive pulmonary toilet with cough assist, airway clearance, and chest physiotherapy.

e. Pain management should limit use of narcotics, which can further suppress adequate cough and recovery. 
6. Pulmonary testing should also be considered during acute disease decompensations with exacerbated symptoms. If the disease is rapidly progressive and respiratory weakness evident on testing, a pulmonary specialist referral and more frequent monitoring is warranted.

a. Incentive spirometry is commonly used in hospitals but manual compression, glossopharyngeal breathing, and insufflations should also be attempted by a respiratory therapist in hospital or at home during the recovery period.

b. Chest X-ray and computed tomography are recommended in the acute setting to identify diaphragm abnormality, collapsed lungs, aspiration, and any other pulmonary pathology.

\section{Other considerations \\ Altitude}

There is a theoretical concern that mitochondrial disease patients may be at risk of deterioration with high-altitude travel due to associated acute to subacute hypobaric hypoxia and associated physiologic changes. Hypoxia brought on by altitude changes is known to produce oxidative damage in cells, increase generation of reactive oxygen species, inhibit mtDNA transcription and translation, decrease mitochondrial muscle fiber content, and downregulate the electron transport chain and fatty acid oxidation capacity. ${ }^{93-95}$ It is known that mtDNA variations may also play a role in the ability to tolerate rapid changes to higher altitudes and that mitochondrial changes stabilize and decrease or resolve under chronic high-altitude conditions. High-altitude sickness brings a risk of associated cerebral edema, pulmonary hypertension, and retinopathy, although there is no concrete evidence yet that these conditions occur in a higher prevalence in mitochondrial disease patients exposed to altitude changes. Chronic conditions, such as underlying pulmonary and cardiovascular disease, can be exacerbated at high altitudes in any patient.

\section{Recommendations.}

1. Careful consideration and planning are needed for highaltitude travel as patients may be susceptible to medical worsening during these times. Rapid recognition and treatment of complications is important.

2. Due to a lower $\mathrm{pO}_{2}$ during air travel, patients with cardiomyopathy or respiratory weakness need to consider oxygen saturation monitoring en route and potentially have access to supplemental oxygen.

\section{Fatigue}

Fatigue is a common complaint in mitochondrial patients. As mitochondrial diseases can cause a combination of neuromuscular disease and systemic symptoms, there are often a multitude of causes for fatigue and worsening of baseline fatigue. The consensus recommendations for the evaluation of fatigue due to a system-specific issue are outlined in several of the individual sections above and summarized below.

\section{Recommendations.}

1. The evaluation of notable or worsening fatigue in mitochondrial patients should include an evaluation for treatable etiologies including consideration of cardiac insufficiency, anemia, an endocrinopathy (specifically thyroid and adrenal disease), worsening myopathy, pulmonary insufficiency, sleep disorder, nutritional and iron deficiencies, and deconditioning.

\section{Care coordination}

Mitochondrial diseases are complex and chronic. Multiple physician visits and frequent hospitalizations are needed at times in a patient's disease course. The diagnosis carries an emotional, psychosocial, and financial burden for the patient and their family. Additional support and care coordination is routinely needed.

\section{Recommendations.}

1. A social work consult should be considered for resource and support planning and to assess for social risk factors that may impact care.

\section{SUMMARY}

The practice of mitochondrial medicine remains challenging due to a paucity of high-quality evidence to guide clinical patient care and management. The MMS used the Delphi method to generate consensus recommendations based on the current knowledge to date, along with the experience of mitochondrial specialists to help begin the process of standardization of care. We hope that these recommendations also raise awareness of the complete spectrum of symptoms and findings in mitochondrial disease and aid in the diagnosis, management, and monitoring of comorbid conditions.

Consensus guidelines are not meant to apply to all cases or conditions and require clinical judgment when used. Recommendations should be adjusted with advances in the field, data from clinical trials, and evidence from future studies.

Due to the rarity of each individual mitochondrial disease, large-scale clinical trials may remain elusive. Networked centers in the United States, through organizations such as the MMS and the North American Mitochondrial Disease Consortium, supported by patient advocacy groups, may serve as an opportunity to allow for gathering of high-quality evidence. Colleagues in other countries have already formed such networks allowing for systematic phenotyping and analysis of this very complex group of patients. The MMS hopes to continue to foster these relationships to advance education, research, and collaboration in the field.

\section{SUPPLEMENTARY MATERIAL}

Supplementary material is linked to the online version of the paper at http://www.nature.com/gim 


\section{ACKNOWLEDGMENTS}

We would like to extend our gratitude to the Wellcome Trust Centre for Mitochondrial Research at Newcastle University for their original creation of Clinical Care Guidelines for select aspects of Mitochondrial Disease (made available online at http://www. newcastle-mitochondria.com). These were reviewed by the consensus group in preparation of their data summaries. We also extend our thanks to Michio Hirano at Columbia for preparing the hematology data summary.

\section{DISCLOSURE}

The authors declare no conflict of interest.

\section{REFERENCES}

1. Parikh S, Goldstein A, Koenig MK, et al. Practice patterns of mitochondrial disease physicians in North America. Part 1: diagnostic and clinical challenges. Mitochondrion 2014;14:26-33.

2. Parikh S, Goldstein A, Koenig MK, et al. Practice patterns of mitochondrial disease physicians in North America. Part 2: treatment, care and management. Mitochondrion 2013;13:681-687.

3. Parikh S, Goldstein A, Koenig MK, et al. Diagnosis and management of mitochondrial disease: a consensus statement from the Mitochondrial Medicine Society. Genet Med 2015;17:689-701.

4. Pfeffer G, Majamaa K, Turnbull DM, Thorburn D, Chinnery PF. Treatment for mitochondrial disorders. Cochrane Database Syst Rev 2012:CD004426.

5. Pfeffer $G$, Horvath $R$, Klopstock $T$, et al. New treatments for mitochondrial disease — no time to drop our standards. Nat Rev Neurol 2013;9:474-481.

6. Turoff M, Linstone HA. The Delphi Method: Techniques and Applications. Addison-Wesley: Reading, MA, 1975.

7. Kullar PJ, Quail J, Lindsey P, et al. Both mitochondrial DNA and mitonuclear gene mutations cause hearing loss through cochlear dysfunction. Brain 2016;139:e33.

8. Santarelli R, Cama E, Scimemi P, et al. Reply: both mitochondrial DNA and mitonuclear gene mutations cause hearing loss through cochlear dysfunction. Brain 2016;139:e34.

9. Sue CM, Lipsett LJ, Crimmins DS, et al. Cochlear origin of hearing loss in MELAS syndrome. Ann Neurol 1998;43:350-359.

10. Wahbi K, Bougouin W, Behin A, et al. Long-term cardiac prognosis and risk stratification in 260 adults presenting with mitochondrial diseases. Eur Heart J 2015;36:2886-2893.

11. Bates MG, Bourke JP, Giordano C, d'Amati G, Turnbull DM, Taylor RW. Cardiac involvement in mitochondrial DNA disease: clinical spectrum, diagnosis, and management. Eur Heart J 2012;33:3023-3033.

12. El-Hattab AW, Scaglia F. Mitochondrial cardiomyopathies. Front Cardiovasc Med 2016;3:25.

13. Florian A, Ludwig A, Stubbe-Drager B, et al. Characteristic cardiac phenotypes are detected by cardiovascular magnetic resonance in patients with different clinical phenotypes and genotypes of mitochondrial myopathy. J Cardiovasc Magn Reson 2015;17:40.

14. Brunel-Guitton C, Levtova A, Sasarman F. Mitochondrial diseases and cardiomyopathies. Can J Cardiol 2015;31:1360-1376.

15. Barends M, Verschuren L, Morava E, Nesbitt V, Turnbull D, McFarland R. Causes of death in adults with mitochondrial disease. JIMD Rep 2016;26: 103-113.

16. Rahman S, Hall AM. Mitochondrial disease-an important cause of endstage renal failure. Pediatr Nephrol 2013;28:357-361.

17. Karaa A, Goldstein A. The spectrum of clinical presentation, diagnosis, and management of mitochondrial forms of diabetes. Pediatr Diabetes 2015;16:1-9.

18. El-Hattab AW, Emrick LT, Hsu JW, et al. Glucose metabolism derangements in adults with the MELAS m.3243A > G mutation. Mitochondrion 2014;18:63-69.

19. Mazzaccara $C$, lafusco $D$, Liguori $R$, et al. Mitochondrial diabetes in children: seek and you will find it. PLOS One 2012;7:e34956.

20. Schaefer AM, Walker M, Turnbull DM, Taylor RW. Endocrine disorders in mitochondrial disease. Mol Cell Endocrinol 2013;379:2-11.

21. Chow J, Rahman J, Achermann JC, Dattani MT, Rahman S. Mitochondrial disease and endocrine dysfunction. Nat Rev Endocrinol 2017;13:92-104.

22. Ng YS, Feeney C, Schaefer AM, et al. Pseudo-obstruction, stroke, and mitochondrial dysfunction: a lethal combination. Ann Neurol 2016;80:686-692.
23. Kadowaki T, Kadowaki H, Mori $\mathrm{Y}$, et al. A subtype of diabetes mellitus associated with a mutation of mitochondrial DNA. N Engl J Med 1994;330:962-968.

24. Matsuzaki M, Izumi T, Shishikura K, Suzuki H, Hirayama Y. Hypothalamic growth hormone deficiency and supplementary GH therapy in two patients with mitochondrial myopathy, encephalopathy, lactic acidosis and stroke-like episodes. Neuropediatrics 2002;33:271-273.

25. Wilichowski E, Gruters A, Kruse K, et al. Hypoparathyroidism and deafness associated with pleioplasmic large scale rearrangements of the mitochondrial DNA: a clinical and molecular genetic study of four children with Kearns-Sayre syndrome. Pediatr Res 1997;41:193-200.

26. Cohen BH, Chinnery PF, Copeland WC. POLG-related disorders. In: Pagon RA, Bird TD, Dolan CR, Stephens K (eds). GeneReviews. University of Washington: Seattle, WA, 1993.

27. Quade A, Zierz S, Klingmuller D. Endocrine abnormalities in mitochondrial myopathy with external ophthalmoplegia. Clin Investig 1992;70:396-402.

28. Rahman S. Gastrointestinal and hepatic manifestations of mitochondrial disorders. J Inherit Metab Dis 2013;36:659-673.

29. Rudolph CD, Hyman PE, Altschuler SM, et al. Diagnosis and treatment of chronic intestinal pseudo-obstruction in children: report of consensus workshop. J Pediatr Gastroenterol Nutr 1997;24:102-112.

30. Parsons $T$, Weimer $L$, Engelstad $K$, et al. Autonomic symptoms in carriers of the m.3243A > G mitochondrial DNA mutation. Arch Neurol 2010;67: 976-979.

31. Sekino $Y$, Inamori $M$, Yamada $E$, et al. Characteristics of intestinal pseudo-obstruction in patients with mitochondrial diseases. World $J$ Gastroentero/ 2012;18:4557-4562.

32. Williams TB, Daniels M, Puthenveetil G, Chang R, Wang RY, Abdenur JE. Pearson syndrome: unique endocrine manifestations including neonatal diabetes and adrenal insufficiency. Mol Genet Metab 2012;106:104-107.

33. Verny C, Amati-Bonneau P, Letournel F, et al. Mitochondrial DNA A3243G mutation involved in familial diabetes, chronic intestinal pseudo-obstruction and recurrent pancreatitis. Diabetes Metab 2008;34:620-626.

34. Staufner C, Haack TB, Kopke MG, et al. Recurrent acute liver failure due to NBAS deficiency: phenotypic spectrum, disease mechanisms, and therapeutic concepts. J Inherit Metab Dis 2016;39:3-16.

35. Dimmock DP, Dunn JK, Feigenbaum A, et al. Abnormal neurological features predict poor survival and should preclude liver transplantation in patients with deoxyguanosine kinase deficiency. Liver Transp/ 2008;14:1480-1485.

36. Parikh S, Karaa A, Goldstein A, et al. Solid organ transplantation in primary mitochondrial disease: proceed with caution. Mol Genet Metab 2016:118:178-184.

37. McKiernan P, Ball S, Santra $S$, et al. Incidence of primary mitochondrial disease in children younger than 2 years presenting with acute liver failure. J Pediatr Gastroenterol Nutr 2016;63:592-597.

38. Finsterer J. Hematological manifestations of primary mitochondrial disorders. Acta Haematol 2007;118:88-98.

39. DiMauro S, Schon EA. Mitochondrial respiratory-chain diseases. N Eng/ J Med 2003;348:2656-2668.

40. Beier UH, Angelin A, Akimova $T$, et al. Essential role of mitochondrial energy metabolism in Foxp3(+) T-regulatory cell function and allograft survival. FASEB J 2015;29:2315-2326.

41. Maclver NJ, Michalek RD, Rathmell JC. Metabolic regulation of T lymphocytes. Annu Rev Immunol 2013;31:259-283.

42. Perl A, Gergely P Jr, Nagy G, Koncz A, Banki K. Mitochondrial hyperpolarization: a checkpoint of T-cell life, death and autoimmunity. Trends Immunol 2004;25:360-367.

43. Broomfield A, Sweeney MG, Woodward CE, et al. Paediatric single mitochondrial DNA deletion disorders: an overlapping spectrum of disease. J Inherit Metab Dis 2015:38:445-457.

44. Ferreira C, Thompson R, Vernon H. Barth syndrome. In: Pagon RA, Adam $\mathrm{MP}$, Ardinger $\mathrm{HH}$, et al. (eds). GeneReviews. University of Washington: Seattle, WA, 1993.

45. Walker MA, Slate N, Alejos A, et al. Predisposition to infection and SIRS in mitochondrial disorders: 8 years' experience in an academic center. J Allergy Clin Immunol Pract 2014;2:465-468, 468.e1.

46. Klein NP, Aukes L, Lee J, et al. Evaluation of immunization rates and safety among children with inborn errors of metabolism. Pediatrics 2011;127:e1139-46.

47. Shahni R, Cale CM, Anderson G, et al. Signal transducer and activator of transcription 2 deficiency is a novel disorder of mitochondrial fission. Brain 2015;138:2834-2846.

48. Emma $F$, Bertini $E$, Salviati $L$, Montini $G$. Renal involvement in mitochondrial cytopathies. Pediatr Nephrol 2012;27:539-550. 
49. Martin-Hernandez E, Garcia-Silva MT, Vara J, et al. Renal pathology in children with mitochondrial diseases. Pediatr Nephrol 2005;20:1299-1305.

50. Rahman S. Mitochondrial disease and epilepsy. Dev Med Child Neurol 2012;54:397-406.

51. Debray FG, Lambert M, Chevalier I, et al. Long-term outcome and clinical spectrum of 73 pediatric patients with mitochondrial diseases. Pediatrics 2007;119:722-733.

52. Khurana DS, Salganicoff L, Melvin JJ, et al. Epilepsy and respiratory chain defects in children with mitochondrial encephalopathies. Neuropediatrics 2008;39:8-13

53. Lee HF, Chi CS, Tsai CR, Chen CH. Epileptic seizures in infants and children with mitochondrial diseases. Pediatr Neurol 2011:45:169-174.

54. Whittaker RG, Devine HE, Gorman GS, et al. Epilepsy in adults with mitochondrial disease: a cohort study. Ann Neurol 2015;78:949-957.

55. Bindoff LA, Engelsen BA. Mitochondrial diseases and epilepsy. Epilepsia 2012;53(suppl 4):92-97.

56. Besse $A$, Wu P, Bruni $F$, et al. The GABA transaminase, ABAT, is essential for mitochondrial nucleoside metabolism. Cell Metab 2015;21:417-427.

57. Mirza NS, Alfirevic A, Jorgensen A, Marson AG, Pirmohamed M. Metabolic acidosis with topiramate and zonisamide: an assessment of its severity and predictors. Pharmacogenet Genomics 2011;21:297-302.

58. DiMauro S, Hirano M. MELAS. In: Pagon RA, Adam MP, Ardinger HH, et al.(eds). GeneReviews. University of Washington: Seattle, WA, 1993

59. El-Hattab AW, Adesina AM, Jones J, Scaglia F. MELAS syndrome: clinical manifestations, pathogenesis, and treatment options. Mol Genet Metab 2015;116:4-12.

60. Altmann J, Buchner B, Nadaj-Pakleza A, et al. Expanded phenotypic spectrum of the m.8344A>G "MERRF" mutation: data from the German mitoNET registry. J Neurol 2016;263:961-972.

61. Seremet OC, Barbuceanu F, Ionica FE, et al. Oral toxicity study of certain plant extracts containing pyrrolizidine alkaloids. Rom J Morphol Embryol 2016;57:1017-1023.

62. Martikainen MH, Ng YS, Gorman GS, et al. Clinical, genetic, and radiological features of extrapyramidal movement disorders in mitochondrial disease. JAMA Neurol 2016;73:668-674.

63. Tranchant $C$, Anheim M. Movement disorders in mitochondrial diseases. Rev Neurol (Paris) 2016;172:524-529.

64. Quinzii CM, Hirano M. Coenzyme Q and mitochondrial disease. Dev Disabil Res Rev 2010;16:183-188.

65. Davidzon G, Greene $P$, Mancuso $M$, et al. Early-onset familial parkinsonism due to POLG mutations. Ann Neurol 2006;59:859-862.

66. Hudson G, Chinnery PF. Mitochondrial DNA polymerase-gamma and human disease. Hum Mol Genet 2006:15(spec no 2):R244-52.

67. Girlanda P, Toscano A, Nicolosi C, et al. Electrophysiological study of neuromuscular system involvement in mitochondrial cytopathy. Clin Neurophysiol 1999;110:1284-1289.

68. Mancuso M, Piazza S, Volpi L, et al. Nerve and muscle involvement in mitochondrial disorders: an electrophysiological study. Neurol Sci 2012;33:449-452

69. Luigetti M, Sauchelli D, Primiano G, et al. Mitochondrial neuropathy: considerations on pathogenesis. Eur J Neurol 2016;23:e55.

70. Mancuso M, Orsucci D, Angelini C, et al. "Mitochondrial neuropathies": A survey from the large cohort of the Italian Network. Neuromuscul Disord 2016;26:272-276

71. Menezes MP, Rahman S, Bhattacharya K, et al. Neurophysiological profile of peripheral neuropathy associated with childhood mitochondrial disease. Mitochondrion 2016:30:162-167.

72. Koenig MK, Emrick L, Karaa A, et al. Recommendations for the management of strokelike episodes in patients with mitochondrial encephalomyopathy, lactic acidosis, and strokelike episodes. JAMA Neurol 2016;73:591-594.

73. Sofou K, De Coo IF, Isohanni $\mathrm{P}$, et al. A multicenter study on Leigh syndrome: disease course and predictors of survival. Orphanet J Rare Dis 2014;9:52
74. Gronlund MA, Honarvar AK, Andersson $S$, et al. Ophthalmological findings in children and young adults with genetically verified mitochondrial disease. Br J Ophthalmol 2010;94:121-127.

75. Zhu CC, Traboulsi El, Parikh S. Ophthalmological findings in 74 patients with mitochondrial disease. Ophthalmic Genet 2017;38:67-69.

76. Yu-Wai-Man P, Chinnery PF. Leber hereditary optic neuropathy. In: Pagon RA, Adam MP, Ardinger HH, et al. (eds). GeneReviews. University of Washington: Seattle, WA, 1993.

77. Klopstock T, Metz G, Yu-Wai-Man P, et al. Persistence of the treatment effect of idebenone in Leber's hereditary optic neuropathy. Brain 2013; 136:e230

78. Klopstock T, Yu-Wai-Man P, Dimitriadis K, et al. A randomized placebocontrolled trial of idebenone in Leber's hereditary optic neuropathy. Brain 2011;134:2677-2686

79. Annaiah TK, Kodakkattil S, Sriemevan A. Pregnancy with mitochondrial encephalopathy lactic acidosis and stroke-like episodes (MELAS syndrome) leading to confusion in the diagnosis of pulmonary embolism. J Obstet Gynaecol 2007;27:618-619.

80. Moriarty KT, McFarland $R$, Whittaker $R$, et al. Pre-eclampsia and magnesium toxicity with therapeutic plasma level in a woman with m.3243A > G MELAS mutation. J Obstet Gynaecol 2008;28:349.

81. Kokawa N, Ishii $Y$, Yamoto M, Nakano R. Pregnancy and delivery complicated by mitochondrial myopathy, encephalopathy, lactic acidosis, and stroke-like episodes. Obstet Gynecol 1998;91:865

82. Yanagawa T, Sakaguchi H, Nakao T, et al. Mitochondrial myopathy, encephalopathy, lactic acidosis, and stroke-like episodes with deterioration during pregnancy. Intern Med 1998;37:780-783.

83. Kovilam OP, Cahill W, Siddiqi TA. Pregnancy with mitochondrial encephalopathy, lactic acidosis, and strokelike episodes syndrome. Obstet Gynecol 1999;93:853.

84. de Laat P, Fleuren LH, Bekker MN, Smeitink JA, Janssen MC. Obstetric complications in carriers of the m.3243A>G mutation, a retrospective cohort study on maternal and fetal outcome. Mitochondrion 2015;25: 98-103.

85. Riley LG, Rudinger-Thirion J, Schmitz-Abe K, et al. LARS2 variants associated with hydrops, lactic acidosis, sideroblastic anemia, and multisystem failure. JIMD Rep 2016;28:49-57.

86. Mancuso M, Orsucci D, lenco EC, Pini E, Choub A, Siciliano G. Psychiatric involvement in adult patients with mitochondrial disease. Neurol Sci 2013;34:71-74.

87. Fattal O, Link J, Quinn $\mathrm{K}$, Cohen $\mathrm{BH}$, Franco K. Psychiatric comorbidity in 36 adults with mitochondrial cytopathies. CNS Spectr 2007;12:429-438.

88. Inczedy-Farkas $G$, Remenyi $V$, Gal $A$, et al. Psychiatric symptoms of patients with primary mitochondrial DNA disorders. Behav Brain Funct 2012:8:9.

89. Byrne E, Dennett X, Trounce I, Burdon J. Mitochondrial myoneuropathy with respiratory failure and myoclonic epilepsy. A case report with biochemical studies. J Neurol Sci 1985;71:273-281.

90. Cros D, Palliyath S, DiMauro S, Ramirez C, Shamsnia M, Wizer B. Respiratory failure revealing mitochondrial myopathy in adults. Chest 1992;101:824-828.

91. Wolfe LF, Joyce NC, McDonald CM, Benditt JO, Finder J. Management of pulmonary complications in neuromuscular disease. Phys Med Rehabil Clin N Am 2012;23:829-853.

92. Ramezani RJ, Stacpoole PW. Sleep disorders associated with primary mitochondrial diseases. J Clin Sleep Med 2014;10:1233-1239.

93. Luo $Y$, Yang $X$, Gao Y. Mitochondrial DNA response to high altitude: a new perspective on high-altitude adaptation. Mitochondrial DNA 2013:24:313-319

94. Hoppeler H, Vogt $M$, Weibel ER, Fluck M. Response of skeletal muscle mitochondria to hypoxia. Exp Physio/ 2003:88:109-119.

95. Murray AJ, Horscroft JA. Mitochondrial function at extreme high altitude. J Physiol 2016;594:1137-1149. 